\title{
Parastomal Hernia-the Achilles Heel of a Permanent Colostomy
}

\author{
Suk-Hwan Lee \\ Department of Surgery, Kyung Hee University School of Medicine, Seoul, Korea
}

\section{See Article on Page 174-179}

The real incidence of parastomal hernia is not well reported, but we assume that it will be much higher than really encountered in clinical practice. A recent prospective study reported a 33\% incidence of parastomal hernia [1]. The majority of parastomal hernias are asymptomatic, and only $10 \%$ of the patients require surgery.

The optimal method for repairing a parastomal hernia has been a debate among surgeons because of the high incidence of recurrence and difficulty in preventing morbidity after the repair. The paper titled "Surgical Treatment of Parastomal Hernia" in this issue of our journal addressed changing trends in the surgical treatment of parastomal hernias. Direct repair and relocation of the stoma were the main surgical strategies of early 90 s whereas mesh repair was the newer surgical approach of the late 90s. These treatments were used to repair not only parastomal hernias but also ventral and inguinal hernias. The socalled "tension free repair" of the hernia is the recent standard for hernia repair.

Theoretically, a mesh repair either synthetic or biologic is superior to the direct repair or relocation of the stoma. Direct repair with the weakened tissues around the stoma is accompanied by the recurrence of the parastomal hernia. This approach is less invasive compare to the relocation of the stoma because we can avoid a laparotomy for the relocation of stoma. However, the application of synthetic mesh around the colostomy is not accepted very well among colorectal surgeons because of the fear of infection. This trend is apparent shown in

Correspondence to: Suk-Hwan Lee, M.D.

Department of Surgery, Kyung Hee University Hostpital at Gangdong, Kyunghee University School of Medicine, 149 Sangil-dong, Gangdong-gu, Seoul 134-727, Korea

Tel: +82-2-440-6134, Fax: +82-2-440-6295

E-mail: leeshdr@khu.ac.kr

(C) 2011 The Korean Society of Coloproctology

This is an open-access article distributed under the terms of the Creative Commons Attribution NonCommercial License (http://creativecommons.org/licenses/by-nc/3.0) which permits unrestricted noncommercial use, distribution, and reproduction in any medium, provided the original work is properly cited. the paper; the authors did not use mesh repair for emergency cases. Although the probability of emergency surgery to repair a parastomal hernia is low, we should define the best treatment option for such an occasion, mesh or no mesh.

With the development of bi-layer synthetic meshes and antibiotics, we now used more synthetic meshes to repair parastomal hernias. Biologic meshes which are known to be resistant to infections are used more commonly in the western countries, but they are rarely used in Korea due to their high cost $[2,3]$. Recently published review papers reported a general trend of lower recurrence rates with the use of synthetic meshes or biologic meshes $[4,5]$. The rate of surgical infection with synthetic meshes is around $10 \%$, and the infections could be managed with antibiotics. However, there is no general consensus about the surgical techniques for mesh placement, and the follow-up period for mesh repair in the literature is relatively to short to allow solid conclusions.

Laparoscopic approaches were introduced more recently [68]. Although a meta-analysis did not find any difference in recurrence rate between the laparoscopic approach and the open approach [7], the minimally invasive procedure should be considered first. We, colorectal surgeons, have a duty to define the right indications for the right patients.

The reported recurrence rate for mesh repair is $11.8 \%$, and the follow-up period for the mesh repair group was about 30 months. These are very excellent results and reflect the enthusiasm of the authors for parastomal hernia repair. Thus, I would congratulate the superb results of the paper. If a more solid conclusion on the optimal method for surgical repair of a parastomal hernia repair is to be reached, nation-wide randomized clinical trials are necessary. I hope members of the Korean Society of Coloproctology will be able to begin such a meaningful trial in the near future.

\section{REFERENCES}

1. Pilgrim $\mathrm{CH}$, McIntyre R, Bailey M. Prospective audit of parastomal hernia: prevalence and associated comorbidities. Dis Colon Rectum 2010;53:71-6.

2. Loganathan A, Ainslie WG, Wedgwood KR. Initial evaluation of 
Permacol bioprosthesis for the repair of complex incisional and parastomal hernias. Surgeon 2010;8:202-5.

3. Slater NJ, Hansson BM, Buyne OR, Hendriks T, Bleichrodt RP. Repair of parastomal hernias with biologic grafts: a systematic review. J Gastrointest Surg 2011;15:1252-8.

4. Tam KW, Wei PL, Kuo LJ, Wu CH. Systematic review of the use of a mesh to prevent parastomal hernia. World J Surg 2010;34: 2723-9.

5. Wijeyekoon SP, Gurusamy K, El-Gendy K, Chan CL. Prevention of parastomal herniation with biologic/composite prosthetic mesh: a systematic review and meta-analysis of randomized controlled trials. J Am Coll Surg 2010;211:637-45.

6. Hiranyakas A, Ho YH. Laparoscopic parastomal hernia repair. Dis Colon Rectum 2010;53:1334-6.

7. Sauerland S, Walgenbach M, Habermalz B, Seiler CM, Miserez M. Laparoscopic versus open surgical techniques for ventral or incisional hernia repair. Cochrane Database Syst Rev 2011;(3): CD007781.

8. Wara P, Andersen LM. Long-term follow-up of laparoscopic repair of parastomal hernia using a bilayer mesh with a slit. Surg Endosc 2011;25:526-30. 